\title{
Prevalence of uremic pruritus in chronic kidney patients undergoing maintenance hemodialysis at Kathmandu Medical College Teaching Hospital
}

\author{
Adhikari L', Kayastha $\mathrm{R}^{2}$, Bhatta $\mathrm{K}^{3}$
}

${ }^{1}$ Laxman Adhikari, Associate Professor; ${ }^{2}$ Rajat Kayastha, Lecturer; ${ }^{3}$ Karuna Bhatta, Resident; Department of Medicine, Kathmandu Medical College Teaching Hospital.

\begin{abstract}
Background: Uremic pruritus is a common and troublesome symptom of chronic kidney disease. It is one of the dominant dermatological manifestations of chronic kidney disease which affects the quality of life of patients.

Objective: To assess the prevalence and severity of uremic pruritus in chronic kidney disease patients undergoing maintenance hemodialysis.

Methodology: A prospective cross-sectional study was done over three months among chronic kidney patients undergoing maintenance hemodialysis to show the association of mineral bone disorders with uremic pruritus. Patients complaining of pruritus were taken as cases and then pruritus was quantified using a Visual Analogue Scale.

Results: 144 patients participated in this study, out of which $41(28.47 \%)$ were females. Mean age was $54.97 \pm 9.75$ years and $68.06 \%$ patients were on dialysis. Uremic pruritus was seen in 80 patients $(55.56 \%)$, amongst them 23 patients ( 28.75 $\%$ ) had severe pruritus as measured by Visual Analogue Scale.Uremic pruritus was more frequent and more severe in patients having higher serum phosphorus levels ( $p=0.004)$.

Conclusion: This study further substantiates the need of adequate control of phosphorus levels in hemodialysis patients to reduce complications like uremic pruritus.
\end{abstract}

Key words: Hemodialysis, Phosphorus, Uremic pruritus

\section{INTRODUCTION}

$\mathrm{P}$ ruritus is a common and troublesome symptom of chronic kidney disease (CKD). It is also known as CKD associated pruritus (CKD-aP). It is one of the dominant dermatological manifestation of CKD, others being xerosis, hyperpigmentation, uremic roseola, calcinosis cutis, half and half nails, impaired homeostasis (ecchymosis and hematoma). Pathophysiology of uremic pruritus (UP) is incompletely understood, but various theories have been given like dry skin, nutritional deficiencies, involvement of inflammatory cytokines, deposition of calcium phosphate crystals in skin, inadequate dialysis resulting into deposition of ammonia and other nitrogenous compounds in skin. Because of this uncertain etiology, UP is very difficult to manage and hence affects the daily activities of the

Address for correspondence

Dr. Rajat Kayastha

Lecturer, Department of Medicine,

Kathmandu Medical College and Teaching Hospital

Sinamangal, Kathmandu patient, including sleep which often results into the patient going into depression.

The prevalence of UP in CKD patients in Nepal is estimated to be $40 \%{ }^{1}$. A global study reported a $42 \%$ prevalence of moderate or extreme UP, which was strongly associated with sleep disturbance, depression, impaired quality of life and mortality ${ }^{2}$. Studies have shown that prevalence of UP increases as CKD progresses and once it starts, it persists. UP is not associated with the start of dialysis, be it hemodialysis or peritoneal dialysis, but some patients complain of noticing it after initiation of dialysis.

UP maybe localized or generalized; generalized UP being more common. Pruritus in dialysis patients is most commonly localized to the back, followed by the forearm with an arterio-venous fistula (perhaps due to frequent washing and traumatization of this region), abdomen, or head ${ }^{3}$. Many studies have suggested that the patient's age, sex, cause of renal failure, type of dialysis etc. have no influence on UP but some studies 
have shown improvement in UP after intensifying the dialysis regimen or by using more biocompatible highflux dialyzers ${ }^{4}$.

As renal function starts to decline in CKD patients, there is a progressive deterioration in kidney's ability to maintain mineral homeostasis, resulting in disruption of normal serum concentrations of phosphorus and calcium. This ultimately results in changes in circulating levels of hormones especially parathyroid hormone (PTH), Vitamin D and its metabolites, fibroblast growth factor-23 (FGF-23) etc. The kidney fails to respond adequately to PTH, thus causing reduced phosphaturia and this phosphate binds with calcium and is deposited as crystals in the skin causing pruritus.

This study was conducted to know the prevalence and severity of UP in CKD patients undergoing maintenance hemodialysis at Kathmandu Medical College Teaching Hospital (KMCTH). It was also done to study the relationship of calcium, phosphorus and parathyroid hormone (PTH) with pruritus. VAS (Visual Analogue Scale) was used for assessment of severity of UP. Serum calcium, phosphorus and PTH were also measured.

\section{METHODOLOGY}

This was a prospective cross-sectional study conducted over a period of three months, from $1^{\text {st }}$ February 2017 to $30^{\text {th }}$ April 2017, among patients receiving maintenance hemodialysis (i.e. four hour sessions, three times a week) for more than three months. Purposive sampling technique was used for selection of cases. Patients undergoing hemodialysis were asked for complaints of pruritus, and those complaining of pruritus were shown a VAS to quantify the severity of pruritus, 0 being no itch while 10 being maximum itch. Patients having dermatologic diseases, exposure/allergy to chemicals and dialyzers were excluded from the study. Patients complaining of pruritus were further investigated.

Blood samples were taken from all patients for assessment of serum levels of calcium, phosphate, iPTH (Intact Parathyroid Hormone). Serum calcium and phosphate was measured by compleximetric method using SelectraProM, while iPTH was measured by chemiluminescence immunoassay (CLIA) method using SnibeMaglumi 1000 . Then these data were entered in software Statistical Package for the Social Sciences
(SPSS) version 23 and the results were produced after analyzing the data. Descriptive data are represented as mean \pm standard deviation (range). Student's t-test was used to compare the means between patients with pruritus and without pruritus, while Pearson correlation coefficient was used to show the relationship between quantitative variables. The level of significance was considered as $\mathrm{P}<0.05$. All the investigations sent were part of management guidelines and no refund or remuneration was given to the patients for participation in the study. All the data were collected after written informed consent by the patient.

\section{RESULTS}

144 CKD patients undergoing maintenance hemodialysis at KMCTH participated in this study, out of which 41 (28.47\%) were female. Mean age of the patients enrolled in the study was $54.97 \pm 9.75$ years, and most of them, 98 patients $(68.06 \%)$ were under maintenance hemodialysis for $>2$ years. In total 80 patients $(55.56 \%)$ complained of UP, and amongst them 46 (57.5\%) had moderate to severe pruritus. It was also observed that patients under maintenance hemodialysis for more than 24 months had UP more than the other group $(p=0.04)$. The demographic and laboratory data of patients suffering from UP compared to those not suffering from UP are presented in Table 1.

Mean age of patients, gender, serum calcium level, and serum parathyroid hormone level showed no significant difference between those having UP compared to those not having UP. The only variable that showed significant difference between the two groups was serum phosphate level $(p=0.03)$. Relation of different variables with the severity of pruritus is shown in Table 2 .

It is observed that 34 patients had milder form of pruritus, but moderate to severe pruritus was more common $(57.5 \%$ vs $42.5 \%)$ in the patients. Increase in levels of serum phosphate is associated with increase in severity of pruritus which is statistically significant $(p=0.004)$. As the level of serum phosphate increased there was statistically significant increase in the calciumphosphate product level $(p=0.007)$.

But the study shows no discernible significance in the severity of pruritus with change in levels of serum calcium or parathyroid hormone $(p=0.1$ and $p=0.79$ respectively). 
Table 1: Patients demographic data according to their pruritus status

\begin{tabular}{|c|c|c|c|}
\hline & $\begin{array}{l}\text { Patients without pruritus } \\
\qquad n=64\end{array}$ & $\begin{array}{l}\text { Patients with pruritus } \\
\qquad \mathrm{n}=\mathbf{8 0}\end{array}$ & P value \\
\hline Age & $56.44 \pm 10.05$ & $53.50 \pm 10.05$ & NS \\
\hline \multicolumn{4}{|l|}{ Gender } \\
\hline Male & $44(68.8 \%)$ & $59(73.7 \%)$ & NS \\
\hline Female & $20(31.2 \%)$ & $21(26.3 \%)$ & NS \\
\hline \multicolumn{4}{|l|}{$\begin{array}{l}\text { Hemodialysis } \\
\text { Duration }\end{array}$} \\
\hline$\leq 24$ months & $26(40.62 \%)$ & $20(25 \%)$ & NS \\
\hline$>24$ months & $38(59.38 \%)$ & $60(75 \%)$ & $0.04^{*}$ \\
\hline Serum Calcium(mg/dl) & $7.27 \pm 0.44$ & $7.16 \pm 0.43$ & NS \\
\hline Serum Phosphate (mg/dl) & $6.98 \pm 0.62$ & $7.21 \pm 0.60$ & $0.03^{* *}$ \\
\hline Serum iPTH ${ }^{\dagger}$ & $297.88 \pm 20.6$ & $299.65 \pm 19.96$ & NS \\
\hline Total & 64 & 80 & \\
\hline
\end{tabular}

† Intact Parathyroid Hormone

* ${ }^{* *}$ shows statistically significant results at $\mathrm{p}<0.05$

Table 2: Severity of pruritus and its relationship with different variables

\begin{tabular}{|c|c|c|c|c|}
\hline & VAS $1-3$ & VAS $4-6$ & VAS 7 or more & $P$ value \\
\hline Age (years) & $58.44 \pm 8.16$ & $56.22 \pm 9.81$ & $53.48 \pm 10.00$ & 0.6 \\
\hline Gender (male/female) & $27 / 7$ & $17 / 6$ & $16 / 7$ & 0.4 \\
\hline Serum Calcium (mg/dl) & $7.02 \pm 0.45$ & $7.22 \pm 0.43$ & $7.30 \pm 0.34$ & \multirow{4}{*}{0.10} \\
\hline $6.0-6.9$ & $23.54 \%$ & $21.74 \%$ & $17.39 \%$ & \\
\hline $7.0-7.9$ & $41.17 \%$ & $43.47 \%$ & $52.17 \%$ & \\
\hline $8.0-8.9$ & $35.29 \%$ & $34.79 \%$ & $30.44 \%$ & \\
\hline Serum Phosphate (mg/dl) & $6.74 \pm 0.41$ & $7.29 \pm 0.50$ & $7.83 \pm 0.25$ & \multirow{4}{*}{$0.004^{*}$} \\
\hline $6.0-6.9$ & $35.30 \%$ & $34.78 \%$ & $21.74 \%$ & \\
\hline $7.0-7.9$ & $47.05 \%$ & $52.17 \%$ & $43.48 \%$ & \\
\hline $8.0-8.9$ & $17.65 \%$ & $13.05 \%$ & $34.78 \%$ & \\
\hline Serum iPTH (pg/ml) & $291.44 \pm 23.57$ & $306.43 \pm 10.05$ & $305.00 \pm 17.74$ & \multirow{3}{*}{0.79} \\
\hline $201-300$ & $64.71 \%$ & $52.18 \%$ & $39.13 \%$ & \\
\hline $301-400$ & $35.29 \%$ & $47.82 \%$ & $60.87 \%$ & \\
\hline Calcium-Phosphate & $47.21 \pm 1.59$ & $52.44 \pm 1.73$ & $57.10 \pm 2.08$ & \multirow{3}{*}{$0.007^{* *}$} \\
\hline Product $\left(\mathrm{mg}^{2} / \mathrm{dl}^{2}\right)$ & $58.82 \%$ & $60.87 \%$ & $30.43 \%$ & \\
\hline $\begin{array}{l}43-52 \\
53-62\end{array}$ & $41.18 \%$ & $39.13 \%$ & $69.57 \%$ & \\
\hline Total number of patients & 34 & 23 & 23 & \\
\hline
\end{tabular}

* ${ }^{* *}$ shows statistically significant results at $p<0.05$

\section{DISCUSSION}

This study showed that uremic pruritus in maintenance hemodialysis is a very common problem (55.56\%), which is similar to $25-50 \%$ prevalence rate shown in various studies ${ }^{5,6}$. It was also observed that patients who had been under hemodialysis for $>2$ years have higher rates of pruritus (75\% vs $25 \%$ ) compared to those under
2 years of hemodialysis ${ }^{7}$, which is due to the fact that uremic toxins especially phosphates are not cleared adequately in conventional hemodialysis resulting in accumulation and subsequent complications.

In addition the results of this study also highlighted that increase in serum phosphate levels was associated with 
increase in uremic pruritus which was also suggested by Gamitri et al ${ }^{8}$. This correlation was strongly supported by Dialysis Outcomes and Practice Patterns Study(DOPPS), which also concluded that odds ratio of developing pruritus increased from 1.2 to $1.37(p<0.0001)$ when serum phosphate levels increased from $5.5-6.7 \mathrm{mg} / \mathrm{dl}$ to $>6.7 \mathrm{mg} / \mathrm{dl}^{2}$. The mean calcium-phosphate product in this study was $51.56 \pm 4.52 \mathrm{mg}^{2} / \mathrm{dl}^{2}$ which was closer to the recommended target of $<55 \mathrm{mg}^{2} / \mathrm{dl}^{2}$ by KDOQI .

But the results showing relationship of serum calcium and serum iPTH with severity of pruritus was conflicting. It was seen that increasing levels of serum iPTH was not associated with increase in severity of pruritus which is in sharp contrast to the findings of Jamal et al and Makhloughet al $^{10,11}$. Similarly, despite being low, serum calcium was not found to be associated with severity

\section{REFERENCES}

1. Shrestha P, Mathur M. Dermatologic Manifestations in Chronic Kidney Disease Patients on Hemodialysis. Nepal Journal of Dermatology, Venereology \&Leprology. 2016;12(1):34-40.

2. Pisoni RL, Wikström B, Elder SJ, Akizawa T, Asano $\mathrm{Y}$, Keen $M L$, et al.Pruritus in haemodialysis patients: International results from the Dialysis Outcomes and Practice Patterns Study (DOPPS).Nephrol Dial Transplant. 2006 Dec;21(12):3495-505.

3. Markova A, Lester J, Wang J, Robinson-Bostom L. Diagnosis of common dermopathies in dialysis patients: a review and update. Semin Dial. 2012 Jul;25(4):408-18.

4. Ko MJ,Wu HY, Chen HY, Chiu YL, Hsu SP, Pai MF, et al. Uremic pruritus, dialysis adequacy, and metabolic profiles in hemodialysis patients: a prospective 5-year cohort study.PLoS One. 2013;8(8):e71404.

5. Mettang T. Pruritus in Renal Disease. In: Carstens E, Akiyama T, editors. Itch: Mechanisms and Treatment [Internet]. Boca Raton (FL): CRC Press/Taylor \& Francis; 2014. Chapter 5. Available from: https:// www.ncbi.nlm.nih.gov/books/NBK200918/

6. Mettang T, Kremer AE. Uremic pruritus.Kidney Int. 2015 Apr;87(4):685-91. of pruritus which also differs from the findings of Tajbakhshet al ${ }^{12}$. These varied findings maybe because of the smaller sample size of the study and short duration of study, hence a bigger multicenter study having a longer follow up is recommended which will encompass larger hemodialysis population thus giving more accurate data on pruritus and its associated factors.

In conclusion, we can say that phosphate control is still a burning issue in hemodialysis patients, and is not only related to dialysis adequacy or efficacy, but is also related to development of other complications of CKD. Hence a more stringent control of phosphate, by reducing consumption of phosphate rich foods and use of phosphate binders, is necessary for both patients and nephrologists to reduce unwanted complications in hemodialysis patients.

7. Melo NC, Elias RM, Castro MC, Romao JE Jr, Abensur $\mathrm{H}$. Pruritus in hemodialysis patients: the problem remains. Hemodial Int. 2009 Jan;13(1):38-42.

8. Gatmiri SM, Mahdavi-Mazdeh M, Lessan-Pezeshki $M$, Abbasi M. Uremic pruritus and serum phosphorus level.Acta Med Iran. 2013 Aug;51(7):477-81.

9. National Kidney Foundation. KDOQI Clinical Practice Guidelines for Bone Metabolism and Disease in Chronic Kidney Disease. Am J Kidney Dis. 2012;60(5):850-886.

10. Jamal A, Subramanian PT. Pruritus among EndStage Renal Failure Patients on Hemodialysis. Saudi J Kidney Dis Transpl. 2000;11(2):181-5.

11. Makhlough A, Emadi M, Sedighi O, Khademloo M, Bicmohamadi AR. Relationship between serum intact parathyroid hormone and pruritus in hemodialysis patients. Iranian Journal of Kidney Diseases. 2013;17(1):25-8.

12. Tajbakhsh R, Joshaghani HR, Bayzayi F, Haddad $M$, Qorbani M. Association between pruritus and serum concentrations of parathormone, calcium and phosphorus in hemodialysis patients. Saudi J Kidney Dis Transpl. 2013;24:702-6. 